\title{
Outcome of decompression using a transnasal ileus tube in malignant adhesive bowel obstruction: A retrospective study
}

\author{
XIANG-JUN HAN, FAN ZHAO, HONG-YING SU and KE XU \\ Department of Radiology, The First Hospital of China Medical University, Shenyang, Liaoning 110001, P.R. China
}

Received February 16, 2017; Accepted July 22, 2017

DOI: $10.3892 /$ mco.2017.1382

\begin{abstract}
Malignant adhesive bowel obstruction caused by peritoneal carcinomatosis is a common complication of advanced abdominal malignancies, and surgical treatment provides little benefit. The present study was undertaken to evaluate the decompression efficacy of a transnasal ileus tube under X-ray guidance, with benign adhesive bowel obstruction patients serving as the control group. A total of 21 patients with malignant adhesive bowel obstruction and 60 patients with benign conditions were enrolled between February 2011 and March 2015. All the patients were treated with transnasal ileus intubation under X-ray guidance. A total of 9 of the 21 malignant cases and 44 of the 60 benign cases were successfully treated with transnasal ileus intubation (42.9 vs. $73.3 \%$, respectively; $\mathrm{P}=0.01$ ). Treatment in 8 malignant and 4 benign cases failed due to death, tube discharge, and/or therapy abandonment, all of which contributed to a significant difference between the two groups (38.1 vs. $6.7 \%$, respectively; $\mathrm{P}=0.01$ ). A total of 4 malignant cases and 12 benign adhesion cases received further surgical treatment, the success rate of which was 50 vs. $91.7 \%$, respectively. The rate of successfully treated intubation cases in all resolution patients was similar between the two groups $(81.8 \%$ in the malignant group and $80 \%$ in the benign group; $\mathrm{P}=0.89)$. In conclusion, ileus tube decompression in patients with malignant conditions was associated with a lower success rate and lower further surgical intervention success rate compared with that observed in patients with benign conditions. However, insertion of an ileus tube may successfully cure $\sim 80 \%$ of all resolution patients in both groups; thus, it may be used as a feasible therapy in malignant adhesive bowel obstruction patients, similar to patients with benign obstruction.
\end{abstract}

Correspondence to: $\mathrm{Dr} \mathrm{Ke} \mathrm{Xu}$, Department of Radiology, The First Hospital of China Medical University, 155 North Nanjing Street, Heping, Shenyang, Liaoning 110001, P.R. China

E-mail: xuke1954@sina.com

Key words: ileus tube, intestinal decompression, peritoneal carcinomatosis, malignant bowel obstruction, adhesive bowel obstruction

\section{Introduction}

Malignant adhesive bowel obstruction caused by peritoneal carcinomatosis is a common and severe complication of advanced abdominal malignancy, reportedly occurring in $10-28.4 \%$ of colorectal cancer patients and $5.5-50 \%$ of ovarian cancer patients (1-4). As a result of poor nutrition and multiple levels of bowel involvement, this condition represents a major challenge regarding clinical treatment. Several studies have demonstrated that conservative treatment with total parenteral nutrition is associated with low efficacy and a high mortality risk, whereas surgical treatment is also associated with a poor outcome (5). Consequently, there is a great clinical demand for a therapeutic method with high efficacy and low invasiveness for such patients.

There is substantial evidence suggesting that bowel rest and effective decompression play an important role in the treatment of intestinal obstruction (6), particularly nasointestinal long tube treatment for benign adhesive small bowel obstructions (7). In 2003, a new transnasal ileus tube was developed and used in clinical practice. Compared with a nasogastric tube, the long tube demonstrated excellent decompression efficacy: It was successful in 70-90\% of patients with a benign adhesive small bowel obstruction (8-11), and has been recommended as a first-line therapeutic strategy. However, there are no studies on the efficacy of decompression by transnasal ileus tube for malignant adhesive bowel obstruction, and no studies comparing the outcome of ileus tube decompression between malignant and benign adhesive bowel obstruction patients have been reported to date.

In this case-control study, we retrospectively investigated a series of patients with malignant adhesive bowel obstruction caused by peritoneal carcinomatosis in order to evaluate the decompression efficacy of a transnasal ileus tube. Patients with a benign adhesive bowel obstruction treated by the same methods served as the control group, and the difference in outcome between the two groups was assessed.

\section{Patients and methods}

Patients. Between February 2011 and March 2015, 81 patients (49 men and 32 women) with a mean age of 60.2 years (range, 14-95 years) were retrospectively analyzed at the First Hospital of China Medical University (Shenyang, China). Malignant adhesive bowel obstruction was identified in 21 patients, 
whereas 60 benign adhesive bowel obstruction patients served as the control group. Malignant adhesive bowel obstruction was defined as adhesive bowel obstruction caused by peritoneal abdominal carcinomatosis, whereas benign adhesive bowel obstruction was defined as adhesive bowel obstruction caused by adhesions due to conditions other than malignancy. The diagnostic criteria for intestinal obstruction included abdominal pain, abdominal distention, vomiting, constipation, and a distended large bowel with an air-fluid level on computed tomography. Other patients were excluded from this study for the following conditions: Fecal obstructions $(n=6)$, internal hernia $(n=1)$ and tumor obstructing the colonic lumen $(n=9)$.

At the time of diagnosis of the bowel obstruction, all the patients were treated by fasting, gastric decompression, total parenteral nutrition, inhibition of gastric acid and pancreatic secretion, analgesics and recommend transnasal ileus intubation. After written informed consent for intestinal decompression was obtained, each patient underwent decompression using a transnasal ileus tube (Create Medic, Tokyo, Japan). A $300-\mathrm{cm}$ long hydrophilic ileus $18 \mathrm{~F}$ tube with three chambers and two balloons was inserted into the jejunum through the nasal cavity under X-ray guidance. The anterior balloon is used to inject sterile water to help advance the tube in the bowel, and the posterior balloon is intended for contrast radiography. The tip of the tube bears suction holes on the side and the ileus tube guidewire is $350-\mathrm{cm}$ long.

The intubation was completed under X-ray guidance; the nasopharynx was anesthetized with $1 \%$ Dicaine. A multipurpose catheter (Cordis Corp., Miami Lakes, FL, USA) was inserted through the nose into the duodenum with the help of a 0.035-inch glidewire (Terumo, Tokyo, Japan) and was then exchanged for a 0.035 -inch stiff guidewire (Terumo) that was inserted into the jejunum for a minimum of $20 \mathrm{~cm}$. The ileus tube guidewire was used to prevent the tube from twisting, and the tube was inserted into the jejunum for a minimum of $20 \mathrm{~cm}$ by following the stiff guidewire. After the guidewire was pulled out, $15 \mathrm{ml}$ of sterile water were injected into the anterior balloon and continuous bowel decompression was initiated with a negative pressure bag. The tube was propelled by bowel peristalsis until decompression treatment was completed. If bowel obstructive symptoms were not relieved after continuous decompression for 3 days, surgical treatment, such as partial intestinal resection and anastomosis, enterolysis or stoma creation, was suggested.

The following patient information was reviewed at the time of ileus tube insertion: Age, sex, duration of obstruction, intestinal obstruction type and site, and laboratory data. Other information, such as patient outcome and the intubation treatment time, was also recorded. The duration of obstruction was calculated as the time interval (in days) from the onset of symptoms to ileus intubation; the obstruction site included the jejunum, ileum and colon. The collected laboratory data included white blood cell count, albumin level, potassium ion concentration and sodium ion concentration. The intubation treatment time was calculated as the time interval (in days) from the start of ileus tube insertion to bowel obstruction resolution without surgery. Bowel obstruction resolution was defined as the disappearance of obstructive symptoms with successful tube removal, and patients tolerating an oral diet without the need for additional intravenous nutritional supplementation. The present study was approved by the Research Ethics Committee of our institution.

Statistical analysis. Continuous variables were expressed as the mean \pm standard deviation, and categorical variables were expressed as a number. Differences between categorical or continuous variables were analyzed using the $\chi^{2}$ test or t-test, respectively. Two-tailed P-values of $<0.05$ were considered to indicate statistically significant differences. Statistical analysis was performed using SPSS software, version 16.0 (SPSS Inc., Chicago, IL, USA).

\section{Results}

Patient characteristics. A total of 21 malignant adhesive obstructive bowel patients and 60 benign adhesive obstructive bowel patients treated with an ileus tube were enrolled in the present study; the primary cancer in malignant obstructive bowel patients consisted of gastric cancer in 5 cases, colic and rectal cancer in 11, ovarian cancer in 3 , pancreatic cancer in 1 and urinary bladder cancer in 1 patient; the previous operation in patients with benign bowel obstruction consisted of gastric surgery in 8 cases, small bowel resection in 3, appendectomy in 3, colorectal surgery in 28 , laparotomy for peritonitis in 2 , enterolysis in 2, gynecological surgery in 3 , abdominal aorta surgery in 1 , hepatic surgery in 1 and splenic surgery in 2 patients; other causes included 1 case of pancreatitis and 6 for unknown reasons. The mean patient age was 60.2 years, and the duration of the obstruction was $11.7 \pm 18.6$ days. Bowel obstruction occurred in the jejunum in 27 , ileum in 41 , and colon in 13 patients. A total of 53 patients were successfully treated with an ileus tube, while treatment failed in 28 patients; no gastrointestinal hemorrhage or perforation occurred during treatment. The background information and patient outcomes are summarized in Table I.

Comparison of characteristics and outcome between the two patient groups. The differences in clinical variables between the malignant and the benign groups are summarized in Table II. Statistical analysis revealed that there was a significant difference in the intubation decompression outcome: Of the 21 malignant obstructive bowel patients, 9 were successfully treated with intubation (Fig. 1), yielding a resolution rate of $42.9 \%$; of the 60 benign patients, 44 were successfully treated, yielding a resolution rate of $73.3 \%(\mathrm{P}=0.01)$. The duration of obstruction in the malignant group was longer compared with that in the benign group (18.3 \pm 21.9 vs. $9.4 \pm 16.9$, respectively), but the difference was not statistically significant $(\mathrm{P}=0.06)$. No significant difference was found between the two groups with regard to age, sex, obstruction site, white blood cell count, albumin, potassium and sodium levels (Table II).

In the 53 successfully treated patients, the mean intubation treatment time was 11.2 \pm 7.7 days; there was no significant difference in intubation treatment time between the malignant and benign bowel obstruction patients $(11.8 \pm 8.5$ vs. $10.0 \pm 7.8$ days, respectively; $\mathrm{P}=0.38$ ). In the 28 patients in whom intubation 
Table I. Characteristics and outcome of 81 patients undergoing obstructive bowel decompression.

\begin{tabular}{lc}
\hline Variables & Values $^{\mathrm{a}}$ \\
\hline Age (years) & \\
Mean (range) & $60.2(14-95)$ \\
Gender, (male/female) & $49 / 32$ \\
Obstruction time, days & $11.7 \pm 18.6$ \\
Obstruction type, (malignant /benign) & $21 / 60$ \\
Obstruction site, (jejunum/ileum/colon) & $27 / 41 / 13$ \\
Laboratory data & \\
White blood cell count (x109/l) & $8.1 \pm 4.6$ \\
Albumin (g/l) & $34.3 \pm 6.7$ \\
Potassium (mmol/l) & $4.1 \pm 0.5$ \\
Sodium (mmol/l) & $138.0 \pm 5.4$ \\
Outcome & \\
Success & 53 \\
Intubation treatment time (days) & $11.2 \pm 7.7$ \\
Failure & 28 \\
Death & 6 \\
Subsequent surgery (success/failure) & $13 / 3$ \\
Discharge with tube & 4 \\
Abandoned therapy & 2 \\
\hline
\end{tabular}

a Data are expressed as mean (range), mean \pm standard deviation, or absolute numbers.

failed, further therapy and outcomes are summarized in Table III. In a total of 8 malignant cases and 4 benign adhesive cases, treatment failed due to death, tube discharge or abandonment of therapy, all of which led to a significant difference in intubation outcome between the two groups (38.1 vs. $6.7 \%$, respectively; $\mathrm{P}=0.01$ ).

In the malignant bowel obstruction group, 3 patients succumbed to tumor cachexia, pulmonary embolism and hematemesis during therapy. A total of 3 patients with a limited expected survival time were discharged from the hospital with continuous ileus tube decompression. Only 4 patients received further surgical interventions, with 2 successful cases, yielding an operative success rate of $50 \%$. In the benign adhesive bowel obstruction group, the deceased patients included 1 case with disseminated intravascular coagulation from septic shock, 1 case of respiratory failure and 1 case of bowel necrosis. Of the 12 benign patients who received further surgical intervention, 11 were successfully treated, yielding an operative success rate of $91.7 \%$.

A total of 66 patients were successfully treated following ileus tube insertion or surgical intervention. Of the 11 resolution patients in the malignant group, 9 were successfully intubated (successful intubation resolution in $81.8 \%$ of all successful malignant resolution patients). In the benign adhesive group, $44 / 55$ had successful intubation resolution (80\%). There was no significant difference between the rates of successfully treated intubation cases in all resolution patients between the two groups $(\mathrm{P}=0.89)$. The flow diagram with the treatment and outcome is presented in Fig. 2.

\section{Discussion}

Intestinal obstruction is a clinical condition that is difficult to treat, time-consuming and costly. If treated with surgical intervention, the recommended two-stage operation requires a longer hospital stay and may also be accompanied by anastomotic leakage, postoperative wound infection, recurrent adhesive bowel obstruction and other complications (12-16). As a result of a poor nutritional status and the multilevel obstructions found in malignant adhesive obstructive bowel patients, treatment is more complicated. Only $30-48.9 \%$ of the patients have been successfully treated, as mentioned in the literature (17-19).

Previously, long tube decompression was shown to be successful in $>70 \%$ of the patients with benign adhesive small bowel obstruction (9-11); this procedure has been confirmed to be a simple, safe and minimally invasive approach, and is recommended as the initial treatment for benign adhesive bowel obstruction. Therefore, benign adhesive bowel obstructive patients were used as the control group in the assessment of the intubation decompression efficacy of malignant adhesive bowel obstruction, and compare the outcome between malignant and benign adhesive bowel obstruction cases.

For patients in the present study who suffered from adhesive bowel obstruction, the time from the onset of obstructive symptoms to ileus tube insertion was $11.7 \pm 18.6$ days; this interval was longer in the malignant compared with that in the benign group $(18.3 \pm 21.9$ vs. $9.4 \pm 16.9$ days, respectively). The time from gastric intubation to ileus intubation in malignant bowel patients was longer compared with benign patients. From our perspective, the majority of patients with malignant obstruction underwent painful and costly antitumor therapies; in addition, the unclear efficacy of ileus intubation in the literature and financial difficulties may have prevented these patients from undergoing ileus intubation sooner.

Our results revealed that the successful intubation resolution rate of malignant adhesive bowel obstruction was significantly lower compared with that of benign bowel obstruction (42.9 vs. $73.3 \%$, respectively; $\mathrm{P}=0.01$ ). Poor patient performance status, systemic deterioration, and complications caused by cancer metastasis may significantly affect these results, as these factors lead to patient death prior to further therapy. In the present study, $38.1 \%$ of the patients ( 3 patients died, 3 were discharged with the tube and 2 abandoned therapy) belong to this group; only $6.7 \%$ of benign patients declined further therapy $(\mathrm{P}=0.01)$. These results highlight that patients with malignant bowel obstruction usually have a poor prognosis.

The palliative medical treatment of malignant bowel obstruction consisted of different drugs for controlling the symptoms. The combination of analgesics, antisecretors, antiemetics and glucocorticoids is the preferred treatment according to the guidelines of the National Comprehesive Cancer Network (18). These drugs may reduce water retention in the intestinal lumen, as well as splanchnic blood flow, indirectly decreasing intestinal wall edema and peristalsis. Over $30 \%$ of patients may be treated successfully with drugs $(18,20)$. In our results, the successful intubation resolution rate of patients with malignant bowel obstruction was $42.9 \%$, and there were no intubation-related complications 
Table II. Clinical variables and patient outcome according to obstruction type.

\begin{tabular}{lccc}
\hline Variables & Malignant & Benign & P-value \\
\hline Age (years) & $60.0 \pm 8.8$ & $60.2 \pm 15.5$ & 0.95 \\
Gender (male/female) & $10 / 11$ & $39 / 21$ & 0.16 \\
Obstruction time (days) & $18.3 \pm 21.9$ & $9.4 \pm 16.9$ & 0.06 \\
Obstruction site (jejunum/ileum/colon) & $5 / 10 / 6$ & $22 / 31 / 7$ & 0.17 \\
White blood cell count (x10 $/ \mathrm{l})$ & $7.3 \pm 3.2$ & $8.3 \pm 5.0$ & 0.37 \\
Albumin (g/l) & $32.5 \pm 6.6$ & $34.9 \pm 6.6$ & 0.16 \\
Potassium (mmol/l) & $4.0 \pm 0.6$ & $4.1 \pm 0.5$ & 0.48 \\
Sodium (mmol/l) & $138.1 \pm 4.0$ & $137.9 \pm 5.9$ & 0.93 \\
Outcome (success/failure) & $9 / 12$ & $44 / 16$ & 0.01
\end{tabular}

Data are expressed as mean \pm standard deviation, or absolute numbers.
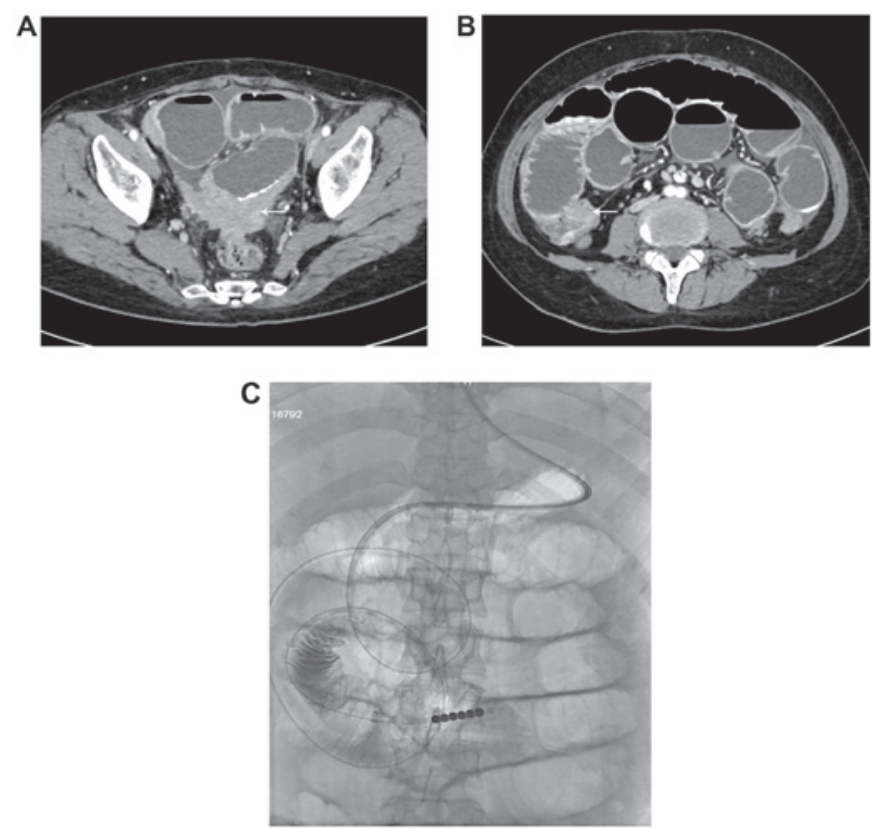

Figure 1. Transnasal ileus tube insertion in malignant adhesive bowel obstruction. (A and B) The patient had undergone surgical treatment for ovarian cancer 3 years prior to presenting with bowel obstruction caused by peritoneal carcinomatosis (arrow). (C) A transnasal ileus tube was inserted under X-ray guidance. The bowel obstruction symptoms resolved after 5 days of continuous decompression.

during the therapy. Our results suggest that the safety and efficacy of ileus tube decompression is comparable to that of other therapies.

A total of 4 malignant adhesive bowel obstruction patients and 12 benign adhesive bowel obstructive patients underwent further surgery after intubation decompression failure, while 2 patients from the malignant and 11 from the benign group were successfully treated. The surgery resolution rate of the malignant obstruction group was significantly lower compared with that of the benign group (50 vs. 91.7\%, respectively). Our results also demonstrated that the outcome of surgical intervention in malignant adhesive bowel obstruction patients is generally poor, and is associated with only a minor clinical benefit, as previously reported $(21,22)$. The multilevel
Table III. Further outcome following intubation decompression failure.

\begin{tabular}{lcc}
\hline $\begin{array}{l}\text { Further } \\
\text { outcome }\end{array}$ & $\begin{array}{c}\text { Malignant } \\
\text { cases (n) }\end{array}$ & $\begin{array}{c}\text { Benign } \\
\text { cases (n) }\end{array}$ \\
\hline Death & 3 & 3 \\
Outpatient with tube & 3 & 1 \\
$\begin{array}{l}\text { Abandoned therapy } \\
\text { Subsequent surgery } \\
\text { (success/failure) }\end{array}$ & $2 / 2$ & 0 \\
\hline
\end{tabular}

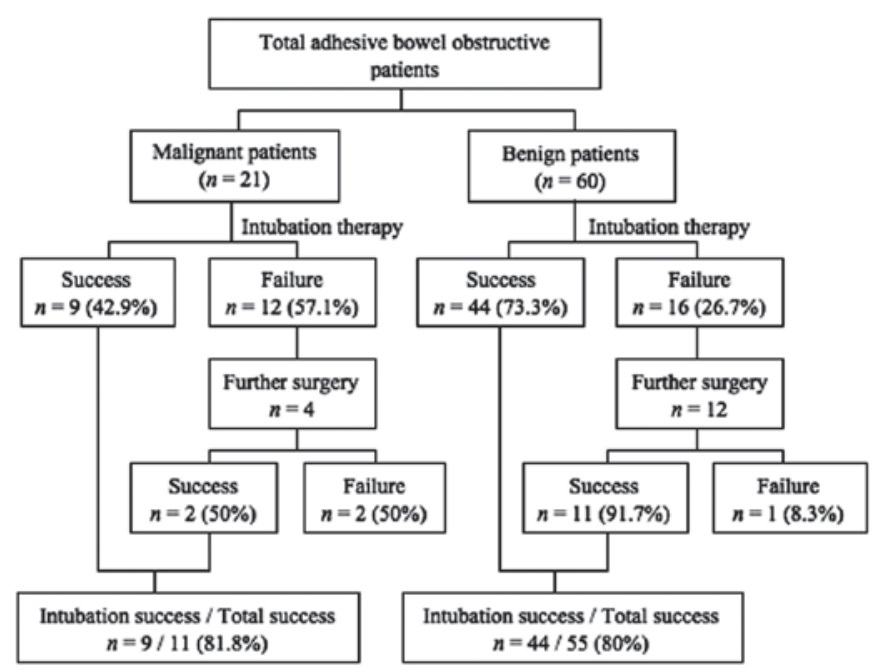

Figure 2. Flow diagram showing the treatment and outcome of 81 patients. A total of 21 malignant and 60 benign cases underwent intubation therapy, with a success rate of 42.9 and $73.3 \%$, respectively.

involvement of the small bowel in the context of peritoneal carcinomatosis, as well as the poor nutritional status, are also important factors. Patients with malignant bowel obstruction should be carefully selected with the aim of improving their quality of life, rather than pursuing emergency surgical procedures (23). 
In the present study, the results demonstrated that there was no significant difference in the total intubation success rate between the two groups ( 81.8 vs. $80 \%, \mathrm{P}=0.89$ ), indicating that ileus intubation may successfully treat $\sim 80 \%$ of all resolution patients in both groups. Therefore, ileus intubation is similar between malignant and benign adhesive bowel obstruction patients. As ileus intubation has been shown to be a feasible first-line therapy for benign adhesive bowel obstructive patients, it may also be useful for relieving malignant adhesive bowel obstruction.

The present study was retrospective, and the small sample size due to the low incidence of malignant bowl obstruction caused by peritoneal carcinomatosis is a major limitation. Therefore, the enrollment of eligible patients occurred over a long period of time. The aim of the present study was to evaluate the efficacy of ileus intubation in malignant and benign adhesive bowel obstruction, and investigate the difference in outcome between the two groups. Therefore, prognostic factors and survival were not investigated, further limiting the interpretation of the results.

In conclusion, malignant adhesive bowel obstruction caused by peritoneal carcinomatosis is a complicated condition; ileus tube decompression in malignant patients is associated with a lower success rate compared with that observed in benign adhesive bowel obstruction patients, and the success of further surgical intervention is also lower in malignant obstruction patients. However, ileus tube decompression in malignant patients may be successful in $\sim 80 \%$ of all resolution cases. Therefore, it is a safe and simple treatment, and insertion of a transnasal ileus tube may be considered as a feasible therapeutic or palliative approach in such cases.

\section{Acknowledgements}

The authors would like to thank the American Journal Experts for the English language editing.

\section{References}

1. Ferguson HJ, Ferguson CI, Speakman J and Ismail T: Management of intestinal obstruction in advanced malignancy. Ann Med Surg (Lond) 4: 264-270, 2015.

2. Ripamonti $\mathrm{C}$ and Bruera $\mathrm{E}$ : Palliative management of malignant bowel obstruction. Int J Gynecol Cancer 12: 135-143, 2002.

3. Feuer DJ, Broadley KE, Shepherd JH and Barton DP: Surgery for the resolution of symptoms in malignant bowel obstruction in advanced gynaecological and gastrointestinal cancer. Cochrane Database Syst Rev: CD002764, 2000.

4. Feuer DJ, Broadley KE, Shepherd JH and Barton DP: Systematic review of surgery in malignant bowel obstruction in advanced gynecological and gastrointestinal cancer. The systematic review steering committee. Gynecol Oncol 75: 313-322, 1999.
5. Chouhan J, Gupta R, Ensor J, Raghav K, Fogelman D, Wolff RA, Fisch M and Overman MJ: Retrospective analysis of systemic chemotherapy and total parenteral nutrition for the treatment of malignant small bowel obstruction. Cancer Med 5: 239-247, 2016.

6. Li D, Du H, Shao G, Xu Y, Li R and Tian Q: Clinical application of transanal ileal tube placement using X-ray monitoring. Oncol Lett 13: 137-140, 2017.

7. Ni Q, Yun L, Liu Z and Shang D: Comparative study of conventional surgery and internal intestinal splinting with long nasointestinal tube in the treatment of acute small bowel obstruction. Hepatogastroenterology 60: 1660-1664, 2013.

8. Chen XL, Ji F, Lin Q, Chen YP, Lin JJ, Ye F, Yu JR and Wu YJ: A prospective randomized trial of transnasal ileus tube vs nasogastric tube for adhesive small bowel obstruction. World J Gastroenterol 18: 1968-1974, 2012.

9. Quatromoni JC, Rosoff L Sr, Halls JM and Yellin AE: Early postoperative small bowel obstruction. Ann Surg 191: 72-74, 1980.

10. Li RH, Li DC, Lv XP and Quan B: Radiophotographically controlled nasointestinal intubation to treat recurrent postoperative adhesive ileus. Cell Biochem Biophys 72: 509-514, 2015.

11. Gowen GF: Long tube decompression is successful in $90 \%$ of patients with adhesive small bowel obstruction. Am J Surg 185: 512-515, 2003.

12. Duron JJ, Silva NJ, du Montcel ST, Berger A, Muscari F, Hennet H, Veyrieres M and Hay JM: Adhesive postoperative small bowel obstruction: Incidence and risk factors of recurrence after surgical treatment: A multicenter prospective study. Ann Surg 244: 750-757, 2006.

13. Torralba JA, Robles R, Parrilla P, Lujan JA, Liron R, Piñero A and Fernandez JA: Subtotal colectomy vs. intraoperative colonic irrigation in the management of obstructed left colon carcinoma. Dis Colon Rectum 41: 18-22, 1998.

14. Deans GT, Krukowski ZH and Irwin ST: Malignant obstruction of the left colon. Br J Surg 81: 1270-1276, 1994.

15. Barillari P, Aurello P, De Angelis R, Valabrega S, Ramacciato G, D'Angelo F and Fegiz G: Management and survival of patients affected with obstructive colorectal cancer. Int Surg 77: 251-255, 1992.

16. Griffith RS: Preoperative evaluation. Medical obstacles to surgery. Cancer 70 (5 Suppl): S1333-S1341, 1992.

17. Anthony T, Baron T, Mercadante S, Green S, Chi D, Cunningham J, Herbst A, Smart E and Krouse RS: Report of the clinical protocol committee: Development of randomized trials for malignant bowel obstruction. J Pain Symptom Manage 34 (Suppl): 49-59, 2007.

18. Tuca A, Guell E, Martinez-Losada E and Codorniu N: Malignant bowel obstruction in advanced cancer patients: Epidemiology, management, and factors influencing spontaneous resolution. Cancer Manag Res 4: 159-169, 2012.

19. Romeo M, de Los LLanos Gil M, Cuadra Urteaga JL, Vilá L, Ahlal S, Indacochea A, Pardo N, Radua J, Font A and Tuca A: Outcome prognostic factors in inoperable malignant bowel obstruction. Support Care Cancer 24: 4577-4586, 2016.

20. Tradounsky G: Palliation of gastrointestinal obstruction. Can Fam Physician 58: 648-652, e317-e321, 2012 (In English, French).

21. Turnbull AD, Guerra J and Starnes HF: Results of surgery for obstructing carcinomatosis of gastrointestinal, pancreatic, or biliary origin. J Clin Oncol 7: 381-386, 1989.

22. van Ooijen B, van der Burg ME, Planting AS, Siersema PD and Wiggers T: Surgical treatment or gastric drainage only for intestinal obstruction in patients with carcinoma of the ovary orperitoneal carcinomatosis of other origin. Surg Gynecol Obstet 176: 469-474, 1993.

23. Furnes B, Svensen R, Helland $H$ and Ovrebo K: Challenges and outcome of surgery for bowel obstruction in women with gynaecologic cancer. Int J Surg 27: 158-164, 2016. 